\title{
Die Verfügbarkeit ambulanter ärztlicher Behandlungen und deren Inanspruchnahme durch über 65jährige in der Schweiz
}

\author{
P. Baschung ${ }^{a}$, A. E. Stuck ${ }^{b, c}$, \\ A. Busato ${ }^{a}$ \\ a Institute for Evaluative Research \\ in Orthopaedic Surgery, \\ Universität Bern \\ b Geriatrische Universitätsklinik, \\ Spital Netz Bern-Ziegler \\ c Geriatrische Abklärungsstation, \\ Klinik für Allgemeine Innere \\ Medizin, Inselspital, Bern
}

\section{Zusammenfassung}

Die vorliegende Studie untersucht die Verfügbarkeit und Inanspruchnahme ambulanter Behandlungen verschiedener medizinischer Fachrichtungen durch Patienten, die älter als 65 Jahre sind. Aus dem Datenpool von santésuisse aus dem Jahr 2004 wurden rund 43 Mio. ambulante Behandlungen von 17290 Leistungserbringern ausgewertet.

In der Schweiz kommt fast jede dritte ambulante Behandlung Menschen über 65 Jahre zugute, obschon der Anteil dieser Altersgruppe in der Gesamtbevölkerung nur bei etwa 16\% liegt. In allen Regionen wird die Mehrzahl der ambulanten Leistungen für ältere Patienten durch Hausärzte erbracht. In ländlichen Regionen gehen jedoch ältere Menschen bedeutend weniger häufig zum Arzt, insbesondere weniger oft zu Spezialärzten als in städtischen Gebieten. Diese Untersuchung zeigt, dass eine geriatrische Ausbildung für alle ambulant tätigen Ärzte wichtig ist. Zudem sind weitere Untersuchungen erforderlich, um festzustellen, ob in ländlichen Gebieten eine Unterversorgung in bezug auf gewisse spezialärztliche Disziplinen besteht (z.B. Gynäkologie, Ophthalmologie).

\section{Einleitung}

Da weltweit der Anteil von Menschen über 65 Jahren stetig steigt [1-7], sehen sich praktisch alle medizinischen Fachrichtungen mit einer zunehmend älteren Population von Patienten konfrontiert. So beträgt beispielsweise in den USA [8] der Anteil von Patienten über 65 Jahren in Hausarztpraxen rund $45,3 \%$.

Untersuchungen aus mehreren Ländern zeigen in bezug auf verschiedene medizinische Fachrichtungen erhebliche Unterschiede zwischen Stadt und Land $[9,10]$. Hausärzte in ländlichen Gegenden der USA erbringen rund zwei Drittel aller ambulanten Dienstleistungen, während in städtischen Gebieten nur etwa ein Drittel der entsprechenden Behandlungen von Haus-

\section{Traitements médicaux}

\section{ambulatoires disponibles et leur}

utilisation par les personnes

âgées de plus de 65 ans en Suisse

La Suisse manque de statistiques différenciées sur le recours aux consultations médicales par les personnes âgées. De ce fait, on peut uniquement supposer l'existence de prises en charge très différenciées des personnes âgées selon les régions. La présente étude recense les prestations médicales ambulatoires à disposition et leur utilisation par les personnes âgées de Suisse. En outre, nous analysons les variations régionales des soins fournis par des médecins de famille et des spécialistes aux patients âgés de plus de 65 ans en Suisse.

Près d'un traitement ambulatoire sur trois est prodigué à des personnes âgées de plus de 65 ans, bien que ce groupe d'âge ne représente que le $16 \%$ environ de la population totale. Dans toutes les régions, la majorité des prestations ambulatoires prodiguées aux personnes âgées le sont par des médecins de famille. Dans les zones rurales, ces personnes vont clairement moins souvent chez le médecin, et notamment moins souvent chez des spécialistes que dans les agglomérations urbaines. La présente étude montre qu'une formation gériatrique est importante pour tous les médecins prodiguant des soins ambulatoires. En outre, d'autres analyses sont nécessaires pour voir s'il ne manque pas certains spécialistes dans les régions rurales (p.ex. gynécologues, ophtalmologues).
Institute for Evaluative Research in Orthopaedic Surgery

Universität Bern

Stauffacherstrasse 78

CH-3014 Bern 
ärzten durchgeführt werden [9]. Die verbleibenden zwei Drittel werden von Spezialärzten erbracht [11].

Für die Schweiz liegen zurzeit kaum entsprechende Zahlen vor. Daher fehlen differenzierte Statistiken über die Inanspruchnahme ärztlicher Konsultationen durch ältere Menschen. Zudem kann nur vermutet werden, dass auch in der Schweiz relevante Stadt-Land-Unterschiede in der Betreuung älterer Menschen bestehen. Für die Gesamtheit aller Patienten ist jedoch bekannt, dass es regionale Unterschiede in bezug auf die Besuche bei Spezialärzten gibt. So werden beispielsweise in der französischsprachigen Schweiz mehr Behandlungen durch Spezialärzte geleistet als in der Deutschschweiz [12]. Deshalb kann angenommen werden, dass in der Westschweiz, unabhängig vom Stadt-Land-Unterschied, auch ältere Menschen generell häufiger durch Spezialärzte behandelt werden als in der deutschsprachigen Schweiz.

Die vorliegende Arbeit untersucht die Verfügbarkeit ambulanter ärztlicher Leistungen und deren Inanspruchnahme durch ältere Menschen in der Schweiz. Zudem analysieren wir die räumliche Variation der spezial- und hausärztlichen Versorgung von Patienten über 65 Jahren in der Schweiz. Dies wird sowohl in bezug auf verschiedene medizinische Fachrichtungen als auch kantonsspezifisch untersucht. Zusätzlich vergleichen wir die ambulanten ärztlichen Leistungen zwischen städtischen und ländlichen Regionen.

\section{Material und Methoden}

Als Datengrundlagen wurden der Datenpool von santésuisse (2004), die Bevölkerungsstatistik und die Raumgliederung des Bundesamtes für Statistik [13] verwendet. Die entsprechende Datenbank wurde im Rahmen des vom OBSAN initiierten und finanzierten Projekts «Démo Médicale» erstellt [14].

Die Daten der santésuisse enthalten das Mengengerüst aller Konsultationen, die 2004 von ambulant tätigen Ärzten zu Lasten der Grundversicherung erbracht wurden. Konsultationen, deren Kosten die Jahresfranchise für den jeweiligen Patienten nicht überstiegen, sind darin nicht enthalten. Der Begriff Konsultation umfasst somit sämtliche Arten von Kontakten zwischen Arzt und Patient, die über die Grundversicherung abgerechnet werden, d.h. Konsultationen in der Praxis, Hausbesuche oder telefonische Konsultationen. Die Daten sind für jeden Leistungserbringer in 20 Altersklassen, nach Geschlecht, nach Gemeindenummer der Patienten sowie nach Leistungsart (Krankheit, Mutter- schaft) klassiert. Leistungen, die im Rahmen von Unfällen anfielen, werden in der vorliegenden Analyse nicht berücksichtigt, da diese meistens nicht über die Grundversicherung, sondern über die Unfallversicherung abgerechnet werden. Neben diesem Mengengerüst enthalten die Daten von santésuisse eine Datei mit Strukturinformationen aller ambulanten Leistungserbringer, die 2004 Leistungen zu Lasten der Grundversicherung abrechneten. Diese Daten umfassen die Anzahl der behandelten Patienten, die Anzahl Lebensjahre der Patienten, die Postleitzahl des Praxisstandortes sowie Angaben zum letzten erworbenen FMH-Facharzttitel. Die Gemeinden wurden gemäss der neunstufigen Gemeindetypologie des BFS [13] eingeteilt. Urbane Zentren, suburbane Gemeinden, einkommensstarke Gemeinden und periurbane Gemeinden wurden als städtische Gemeinden bezeichnet, während touristische Gemeinden, industrielle und tertiäre Gemeinden, ländliche Pendlergemeinden, agrar-gemischte Gemeinden und agrarische Gemeinden als ländliche Gemeinden klassiert wurden. Die über 40 verschiedenen FMH-Facharzttitel wurden gemäss dem Projekt «Démo Médicale» des OBSAN auf der Basis des zuletzt erworbenen FMH-Facharzttitels in sieben Fachrichtungen zusammengefasst [14], d.h., Ärzte mit den Facharzttiteln «Allgemeinmedizin», «Allgemeine Innere Medizin», «Praktische Ärztin oder praktischer Arzt ohne Spezialisierung» sowie «Gruppenpraxen» (nur juristische Personen) werden als Hausärzte bezeichnet. Alle anderen Ärzte wurden in entsprechenden Facharztgruppen zusammengefasst und im Rahmen dieser Arbeit als Spezialärzte bezeichnet (invasiv bzw. nichtinvasiv tätige Fachärzte, Gynäkologie, Psychiatrie, Pädiatrie und Spital ambulant). Ärzte, die 2004 keine Leistungen zu Lasten der Grundversicherung erbrachten, wurden von der Studie ausgeschlossen.

Die Auswertung der Daten erfolgte rein deskriptiv auf zwei Ebenen:

- Stufe der Leistungserbringer: Die Auswertung untersuchte die regionale Verteilung der Leistungserbringer und das entsprechende Mengengerüst der erbrachten Leistungen (d.h. Anzahl Konsultationen pro Gemeindetyp, nach Standort der Praxis);

- Stufe Patient/Konsultationen: Die Auswertung der regionalen Verteilung der beanspruchten Leistungen als jährliche Behandlungsinzidenz pro Gemeinde (d.h. Anzahl Konsultationen/Einwohner pro Gemeindetyp, nach Wohnort der Patienten). 


\section{Resultate}

\section{Leistungserbringer / erbrachte Leistungen} Insgesamt standen Daten von 17290 Leistungserbringern und über 43 Mio. Konsultationen zur Verfügung. Die Untersuchung zeigt, dass 38\% aller ambulanten Leistungserbringer im Jahr 2004 Hausärzte waren $(\mathrm{n}=6564)$, die $49 \%(\mathrm{n}=$ 21475874) aller ambulanten Behandlungen durchführten [14]. Im Jahre 2004 betrug der Anteil ambulanter Behandlungen, die von über 65jährigen (15,8\% der Schweizer Bevölkerung [15]) bezogen wurden, $29,3 \%(n=12762810)$, wobei $60 \%$ dieser Leistungen von Hausärzten erbracht wurden (Tab. 1). Tabelle 2 zeigt die Leistungen der verschiedenen Arztdisziplinen für die drei Altersgruppen Kinder, Erwachsene zwischen 16 und 65 Jahren und ältere Menschen über 65 Jahre. Dabei zeigt sich, dass Hausärzte,

Tabelle 1

Behandlungen von Patienten über 65 Jahre nach Facharztdisziplin.

$\begin{array}{lc}\text { Ärztegruppe } & \begin{array}{c}\text { Anteil der Behandlungen } \\ \text { für Patienten }>65(\%)\end{array} \\ \text { Hausärzte } & 60,37 \\ \text { Spezialärzte } & \\ \text { Invasiv tätige Spezialärzte } & 12,97 \\ \text { Nichtinvasiv tätige Spezialärzte } & 11,92 \\ \text { Gynäkologie } & 1,41 \\ \text { Psychiatrie, Kinderpsychiatrie } & 0,93 \\ \text { Pädiatrie } & 0,05 \\ \text { Nicht klassiert } & 1,20 \\ \text { Spital ambulant } & 11,15 \\ \text { Total } & 100,00\end{array}$

Tabelle 2

Verteilung der ambulanten Behandlungen nach Fachrichtung und Altersgruppen (\%).

$\begin{array}{lccc}\text { Ärztegruppe } & \text { Kinder } & 16-65 & >65 \\ \text { Hausärzte } & 5,45 & 58,67 & 35,88 \\ \text { Spezialärzte } & & & \\ \text { Invasiv tätige Spezialärzte } & 0,36 & 91,54 & 8,10 \\ \text { Nichtinvasiv tätige Spezialärzte } & 3,43 & 63,16 & 33,40 \\ \text { Gynäkologie } & 7,83 & 53,82 & 38,36 \\ \text { Psychiatrie, Kinderpsychiatrie } & 6,04 & 89,45 & 4,51 \\ \text { Pädiatrie } & 93,55 & 6,16 & 0,29 \\ \text { Nicht klassiert } & 4,42 & 69,39 & 26,19 \\ \text { Spital ambulant } & 7,94 & 66,49 & 25,57\end{array}$

nichtinvasiv tätige Spezialärzte und Gynäkologen zwischen 30 und 40\% ihrer Konsultationen für Patienten über 65 erbringen; Psychiater und invasiv tätige Spezialärzte dagegen erbringen weniger als $10 \%$ ihrer Leistungen für ältere Menschen.

Hinsichtlich der geographischen Verteilung von Haus- und Spezialärzten machen Hausärzte in agrarischen Gemeinden rund $75 \%$ aller dortigen ambulant tätigen Ärzte aus, während es in urbanen Zentren lediglich 27,2\% sind. In ländlichen Gebieten ist der Anteil erbrachter ambulanter Behandlungen von Patienten über 65 , gemessen an der Gesamtheit der erbrachten Leistungen, in allen Disziplinen deutlich höher als in städtischen Gebieten (Tab. 3). Zwischen Hausund ambulant tätigen Spitalärzten bestehen in bezug auf den Anteil ambulanter Behandlungen für ältere Patienten beträchtliche Stadt-LandUnterschiede (Abb. 1). In urbanen Zentren erbringen Spezialärzte und ambulante Spitalabteilungen insgesamt mehr ambulante Dienstleistungen für Patienten über 65 als Hausärzte (56,3 vs. 43,7\%). Dieses Phänomen zeigt sich aber nur in Zentren. In allen anderen Gemeindetypen wird die Mehrzahl der ambulanten Leistungen für Menschen über 65 von Hausärzten erbracht. Einen besonders hohen Anteil der ambulanten Leistungen für Menschen über 65 erbringen Hausärzte in periurbanen $(91,3 \%)$, in ländlichen Pendlergemeinden (89,5\%), in agrargemischten $(93,3 \%)$ und in agrarischen Gemeinden (97,9\%) (Abb. 1).

Bezüglich der kantonalen Verteilung ambulanter Behandlungen von älteren Patienten durch den Hausarzt zeigen sich ebenfalls klare Unterschiede (Abb. 2). So werden im Kanton Obwalden rund 82,9\% aller ambulanten Dienstleistungen von Patienten über 65 durch den Hausarzt erbracht, während es im Kanton BaselStadt lediglich $44,2 \%$ sind.

\section{Beanspruchte Leistungen / Behandlungsinzidenz}

60,7\% aller ambulanten ärztlichen Leistungen, die von Menschen über 65 in Anspruch genommen wurden, stammten von Hausärzten, wobei diese Inanspruchnahme ebenfalls deutliche regionale Unterschiede aufweist. In städtischen Gemeinden betrug der Anteil von beanspruchten hausärztlichen Leistungen 57,9\%, während in ländlichen Gemeinden 68,4\% aller Leistungen von Hausärzten stammten.

Insgesamt betrug die Behandlungsinzidenz des Jahres 2004, d. h. die Anzahl Arztkonsultationen pro Einwohner im Alter von 66 bis 80, für alle medizinischen Fachrichtungen 11,2 Kon- 
Abbildung 1

Ambulante Behandlungen von Patienten über 65 Jahre nach Arztdisziplin (Gemeindetyp nach Praxisstandort der Ärzte).

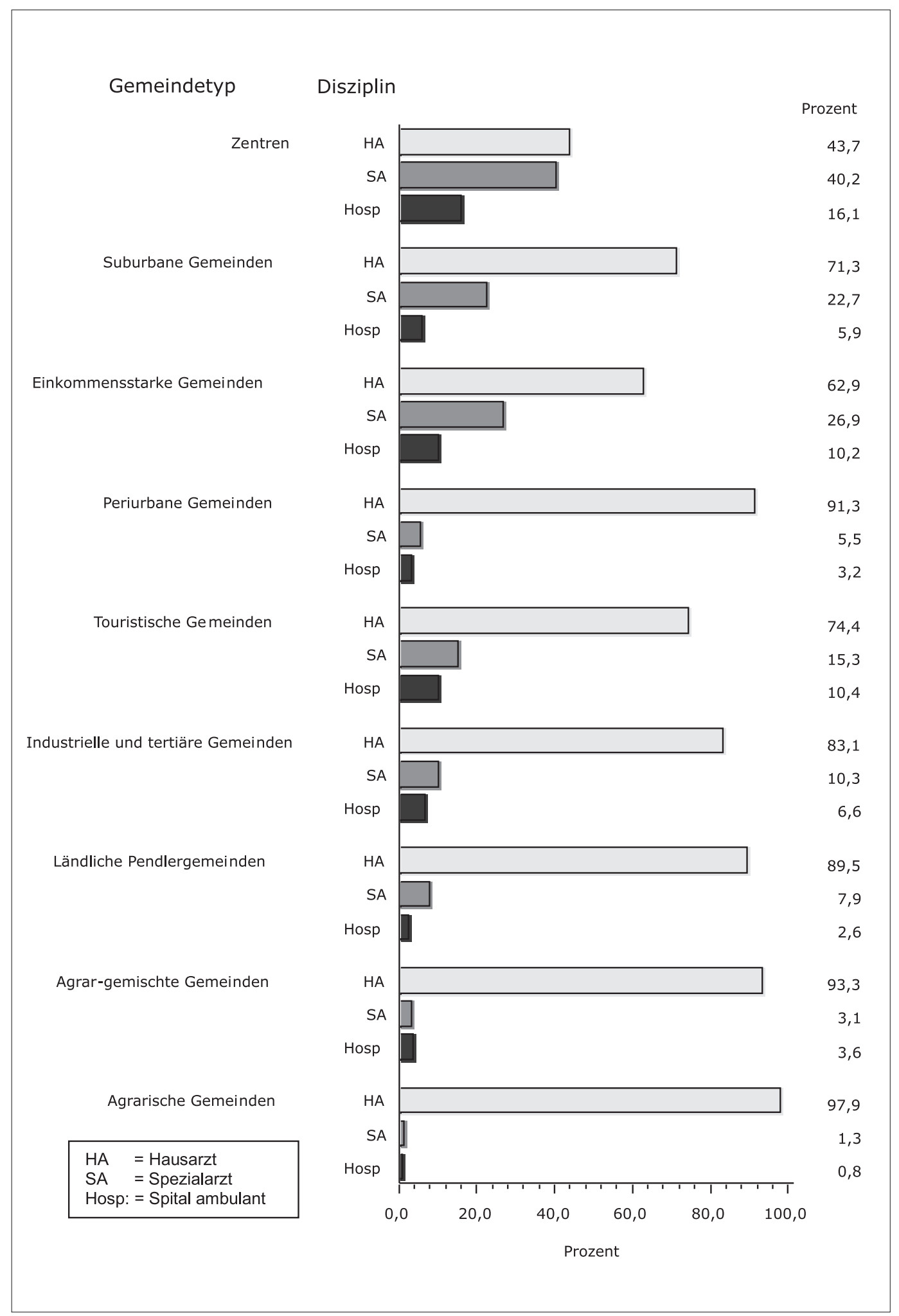


sultationen und für Einwohner über 80 Jahre 15,0 Konsultationen [14]. Die Analyse der StadtLand-Unterschiede zeigt auf, dass Einwohner über 65 Jahre aus städtischen Gebieten durchwegs höhere Behandlungsinzidenzen aufweisen als Einwohner aus ländlichen Gebieten (Tab. 4) wobei die Behandlungsinzidenzen bei Hausärzten für Bewohner aus ländlichen (6,90 Konsultationen) und aus städtischen Regionen (7,34 Kon- sultationen) nahezu gleich sind (Tab. 4, Abb. 3). Die Differenz der Behandlungsinzidenz zwischen der Gesamtheit aller medizinischen Fachrichtungen und der Hausärzte zeigt denn auch, dass die Stadt-Land-Unterschiede weitgehend darauf zurückzuführen sind, dass ältere Menschen aus ländlichen Gegenden weniger oft zum Spezialarzt gehen als in städtischen Gegenden (Tab. 4). Aufgrund der entsprechenden Summen aller

\section{Tabelle 3}

Anteil an ambulanten Behandlungen von Patienten über 65 Jahre gemessen am Total aller medizinischen Konsultationen im jeweiligen Gemeindetyp.

\begin{tabular}{|c|c|c|}
\hline Gemeindetyp & $\begin{array}{l}\text { Anzahl Konsultationen }>65 \\
\text { Anzahl alle Konsultationen }\end{array}$ & $\%$ \\
\hline \multicolumn{3}{|l|}{ Städtische Gemeinden } \\
\hline Zentren & $6453013 / 23076985$ & 27,96 \\
\hline Suburbane Gemeinden & $2840859 / 10054536$ & 28,25 \\
\hline Einkommensstarke Gemeinden & $533902 / 1650544$ & 32,35 \\
\hline Periurbane Gemeinden & $642504 / 2031539$ & 31,63 \\
\hline \multicolumn{3}{|l|}{ Ländliche Gemeinden } \\
\hline Touristische Gemeinden & $377092 / 1041782$ & 36,20 \\
\hline Industrielle und tertiäre Gemeinden & $1171335 / 3484972$ & 33,61 \\
\hline Ländliche Pendlergemeinden & $300765 / 957912$ & 31,40 \\
\hline Agrar-gemischte Gemeinden & $394247 / 1143708$ & 34,47 \\
\hline Agrarische Gemeinden & $47552 / 116734$ & 40,74 \\
\hline Total & $12761269 / 43558712$ & 29,30 \\
\hline
\end{tabular}

Abbildung 2

Ambulante Behandlungen von Patienten über 65 Jahre durch den Hausarzt nach Kanton.

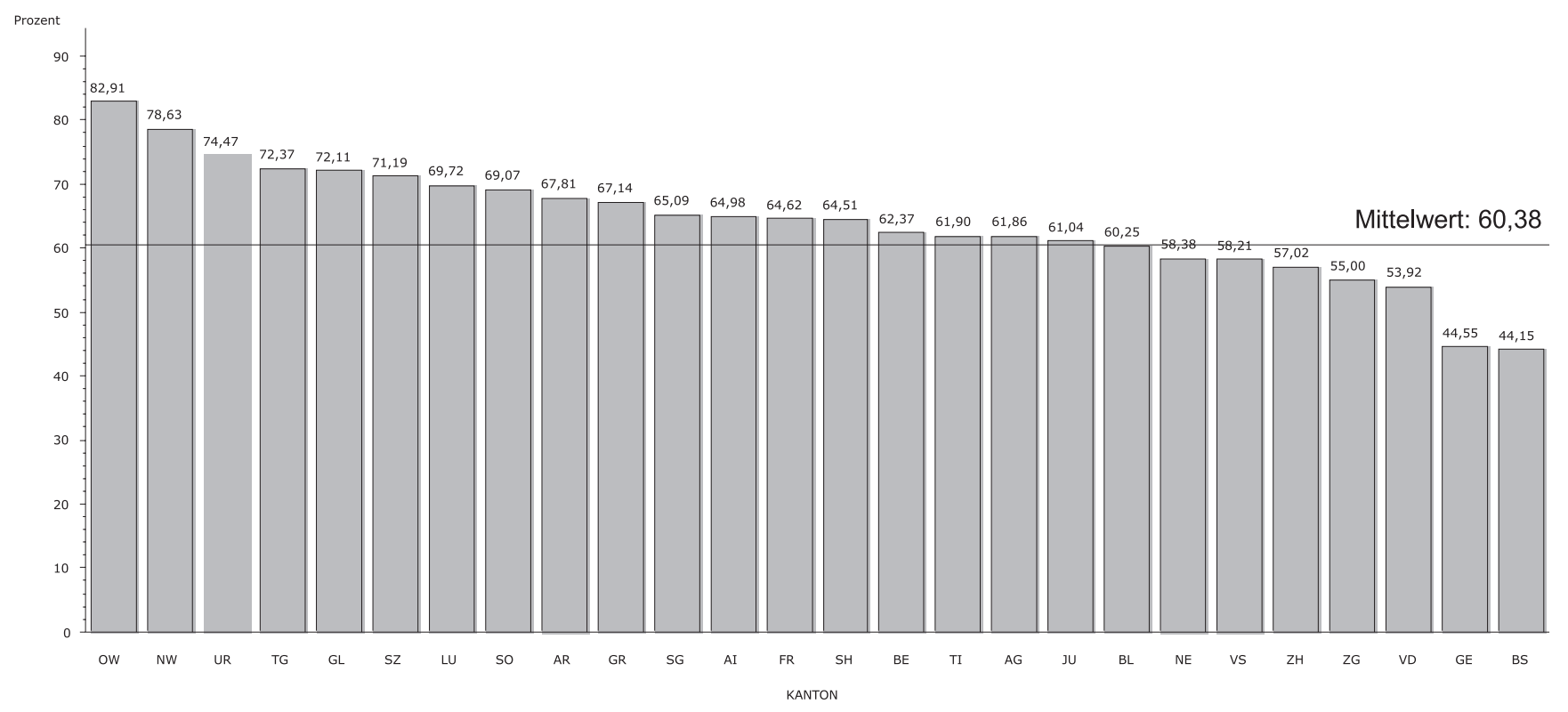


Konsultationen beanspruchen Menschen über 65 Jahre in städtischen Gebieten 2,5 Hausarztbesuche pro Spezialarztbesuch, und in ländlichen Gebieten vergrössert sich dieses Verhältnis auf $4: 1$.

Zur weiteren Analyse der Stadt-LandUnterschiede untersuchten wir zusätzlich die Behandlungshäufigkeit der Einwohner über 65 nach Kategorie von Spezialärzten (Tab. 4). Dabei interessierten insbesondere die Fachbereiche

\section{Abbildung 3}

Behandlungsinzidenzen von Patienten über 65 Jahre nach Arztdisziplin und Gemeindetyp.

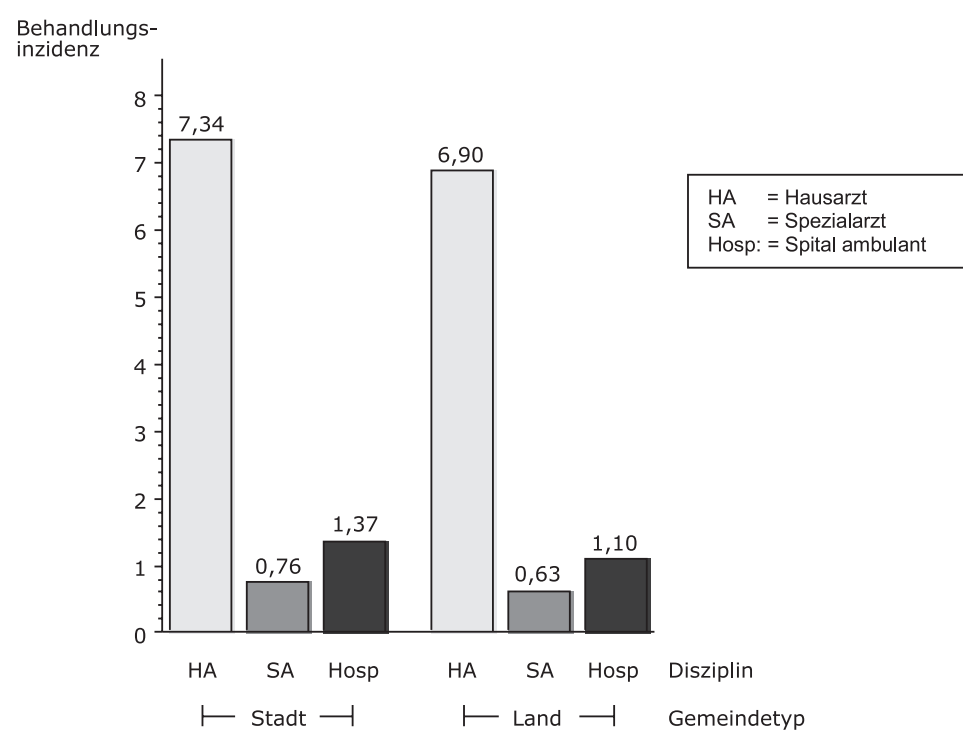

Gynäkologie, Ophthalmologie und Urologie, da diese drei Fachbereiche in der Regel Leistungen erbringen, die von Hausärzten nicht erbracht werden können. Wir stellten folgende Hypothese auf: Eine geringere Inanspruchnahme dieser drei Fachdisziplinen in agrarischen Gemeinden im Vergleich zu urbanen Gemeinden müsste demnach als Indiz für eine spezialärztliche Unterversorgung in agrarischen Gemeinden gewertet werden. Tabelle 4 bestätigt unsere Hypothese, dass sich auch in bezug auf die drei Fachbereiche Stadt-Land-Unterschiede finden. So liegt beispielsweise die Anzahl gynäkologischer Konsultationen in urbanen Zentren bei 0,21 pro Person und Jahr; im Vergleich dazu beträgt die Inzidenz gynäkologischer Behandlungen in agrarischen Gemeinden nur etwa die Hälfte $(0,11$ pro Person und Jahr). In Tabelle 4 sind zudem die Unterschiede der Behandlungsinzidenz für die Gruppe der nichtinvasiv tätigen Spezialärzte auffallend:
In urbanen Zentren beträgt die entsprechende Behandlungsinzidenz 1,66 und in agrarischen Gemeinden nur 0,63 Behandlungen pro Einwohner und Jahr.

\section{Diskussion}

In dieser Studie wird die geographische Verteilung ambulanter Behandlungen von Patienten über 65 Jahre für verschiedene medizinische Fachrichtungen untersucht. Es zeigt sich, dass drei Viertel aller Ärzte in ländlichen Gegenden Hausärzte sind, während diese Ärztegruppe in städtischen Gebieten nur etwa ein Viertel aller ambulant tätigen Ärzte ausmacht. Es ist deshalb nicht erstaunlich, dass in der Schweiz erhebliche Stadt-Land-Unterschiede in der Verteilung ambulanter Behandlungen von Patienten über 65 durch Haus-, Spezial- und Spitalärzte bestehen. Ähnlich wie in den USA $[9,11]$ werden diese Patienten in ländlichen Gebieten vor allem durch Hausärzte behandelt, während in städtischen Gebieten mehr ambulante Leistungen durch Spezialärzte erbracht werden. In der Schweiz treffen diese Unterschiede allerdings nur für den Gemeindetyp der urbanen Zentren zu. Ein Vergleich zu Probst et al. [9], wo nur zwischen Stadt und Land unterschieden wird, ist deshalb ist nur bedingt möglich.

In ländlichen Regionen gehen ältere Menschen weniger häufig zum Arzt. Insbesondere nehmen sie weniger Spezialarztkonsultationen in Anspruch als Menschen in städtischen Regionen. Für diese Unterschiede sind verschiedene Erklärungen möglich. Es könnte sein, dass ältere Menschen auf dem Land einen erschwerten $\mathrm{Zu}-$ gang zu Spezialärzten haben und somit bei älteren Menschen eine Unterversorgung in bezug auf spezialärztliche Behandlung besteht. Alternativ ist es auch denkbar, dass ältere Menschen in der Stadt zum Teil unnötig oft direkt einen Spezialarzt aufsuchen, statt sich an einen Hausarzt zu wenden. Die vorliegende Studie kann hier keine Erklärung liefern - weitere Studien zu dieser Fragestellung sind erforderlich.

Insgesamt erbringen Hausärzte, nichtinvasiv tätige Spezialärzte und Gynäkologen etwa einen Drittel ihrer ambulanten Behandlungen für Menschen über 65 Jahre. Damit bilden diese Behandlungen einen beträchtlichen Anteil der Tätigkeit dieser Ärzte. Die Hausarztmedizin spielt daher eine äusserst wichtige Rolle in der Behandlung von älteren Patienten. Neueste Erhebungen machen deutlich, dass die Alterungsgeschwindigkeit der Schweizer Bevölkerung rapide zunimmt [16]. Aus diesem Grund sollte der geriatrischen Aus-, Weiter- und Fortbildung 
ausreichend Platz eingeräumt werden, damit vor allem zukünftige Hausärzte den steigenden Ansprüchen dieser Altersgruppe bezüglich Behandlungshäufigkeit und -qualität gerecht werden können.

Aufgrund der Tatsache, dass ausser in urbanen Zentren Patienten über 65 vor allem durch Hausärzte betreut werden, kann davon ausgegangen werden, dass die Anzahl derartiger Konsultationen für Hausärzte noch weiter zunehmen wird. Da die Anzahl Hausärzte auf dem Land durch immer weniger attraktive Arbeitsbedingungen und durch die Konkurrenzsituation mit den Spitälern und anderen Leistungserbringern stetig sinkt [17], könnte mittel- bis langfristig eine Unterversorgung entstehen, so dass das Angebot an ambulanten Behandlungen für ältere Patienten durch Hausärzte nicht mehr ausreicht [18]. Als Folge würden die wichtige Triagefunktion der Hausärzte und die im Vergleich zu Spezialärzten bzw. Spitälern kostengünstigen Behandlungen der Hausarztpraxis wegfallen. Dieses Szenario könnte zu einem weiteren Kostenanstieg im Gesundheitswesen führen. Weiter ist mit dem Schwinden der Hausärzte in ländlichen Gebieten auch die Qualität der Notfallversorgung auf dem Land gefährdet, und es bliebe den entsprechenden Populationen nichts anderes übrig, als sich in städtischen Gebieten behandeln zu lassen.

Die kantonalen Unterschiede bezüglich der Verteilung der ambulanten Behandlungen für Patienten über 65 Jahre durch Haus- und Spezialärzte bestätigen andere Untersuchungen [12, 14], die zeigen, dass in den französischsprachigen Kantonen ambulante Behandlungen eher durch Spezial- als durch Hausärzte erbracht werden.

Die Daten der Inanspruchnahme ambulanter Leistungen durch die Bevölkerung weisen darauf hin, dass Bewohner über 65, analog zur Gesamtbevölkerung, in ländlichen Gegenden pro Jahr deutlich weniger oft zum Arzt gehen als die entsprechenden Populationen aus städtischen Gebieten. Die Aufteilung nach medizinischen Fachrichtungen zeigt aber, dass die StadtLand-Unterschiede weitgehend auf unterschiedliche Behandlungsinzidenzen bei Spezialärzten zurückzuführen sind.

In bezug auf die variierende regionale Altersstruktur der Bevölkerung weisen die Behandlungsinzidenzen ein paradoxes Muster auf. Eigentlich sollte auf dem Land ein höherer Bedarf an medizinischen Leistungen sein, da die Population in ländlichen Gegenden im Durchschnitt älter ist als in der Stadt [19]. Weitere Untersuchungen im Rahmen des laufenden Projekts «Démo Médicale» sind deshalb der theoretischen Beschreibung derartiger Phänomene und der Bildung empirischer Modelle gewidmet. Es wird sich zeigen, ob die Nachfrage nach Leistungen durch angebotsseitige Strukturen oder durch effektive Bedürfnisse der Bevölkerung bedingt wird.

\section{Stärken und Schwächen der Studie}

Die grosse Stärke dieser Studie ist der Umfang der Datenbasis. Es handelt sich dabei nicht um eine Stichprobe, sondern um eine Vollerhebung aller

Tabelle 1

Durchschnittliche Anzahl Konsultationen pro Einwohner über 65 pro Jahr nach Gemeindetyp und Arztdisziplin.

\begin{tabular}{|c|c|c|c|c|c|c|c|}
\hline Gemeindetyp & Total & Hausarzt & Spezialarzt & $\begin{array}{l}\text { davon } \\
\text { Gynäkologie }\end{array}$ & $\begin{array}{l}\text { davon } \\
\text { Ophthalmologie }\end{array}$ & $\begin{array}{l}\text { davon } \\
\text { Urologie }\end{array}$ & Spital ambulant \\
\hline \multicolumn{8}{|l|}{ Städtische Gemeinden } \\
\hline Zentren & 12,26 & 7,24 & 3,65 & 0,17 & 0,91 & 0,14 & 1,37 \\
\hline Suburbane Gemeinden & 12,49 & 7,68 & 3,43 & 0,18 & 0,95 & 0,14 & 1,37 \\
\hline Einkommensstarke Gemeinden & 11,32 & 6,10 & 3,82 & 0,21 & 0,88 & 0,18 & 1,44 \\
\hline Periurbane Gemeinden & 11,70 & 7,35 & 3,02 & 0,16 & 0,86 & 0,15 & 1,36 \\
\hline \multicolumn{8}{|l|}{ Ländliche Gemeinden } \\
\hline Touristische Gemeinden & 11,01 & 7,21 & 2,67 & 0,13 & 0,74 & 0,13 & 1,19 \\
\hline Industrielle und tertiäre Gemeinden & 11,00 & 7,57 & 2,32 & 0,12 & 0,75 & 0,10 & 1,13 \\
\hline Ländliche Pendlergemeinden & 10,10 & 6,76 & 2,30 & 0,14 & 0,74 & 0,13 & 1,10 \\
\hline Agrar-gemischte Gemeinden & 10,01 & 6,93 & 2,05 & 0,11 & 0,72 & 0,12 & 1,09 \\
\hline Agrarische Gemeinden & 8,72 & 6,14 & 1,76 & 0,15 & 0,67 & 0,15 & 1,05 \\
\hline Gesamte Schweiz & 12,12 & 7,32 & 3,45 & 0,17 & 0,92 & 0,13 & 1,35 \\
\hline
\end{tabular}


ambulanten Leistungen, die 2004 in der Schweiz zu Lasten der obligatorischen Grundversicherung erbracht wurden. Daraus ergibt sich eine sehr hohe Generalisierbarkeit. Eine Limitierung dieser Studie ist, dass unfallbedingte Konsultationen nicht eingeschlossen sind. Ebenfalls von Nachteil ist, dass anhand der vorliegenden Daten nicht ersichtlich ist, welche Art Leistung ein Arzt erbracht hat, da lediglich seine Spezialisierung bzw. sein letzter erworbener FMH-Facharzttitel bekannt sind. Es kann demnach beispielsweise nicht ausgeschlossen werden, dass hausarztmedizinische Leistungen auch durch Spezialärzte erbracht wurden und umgekehrt. Weiter kann anhand der hier verwendeten Gemeindetypologie des Bundesamtes für Statistik [13] keine direkte Einteilung in Stadt und Land erfolgen, weshalb ein Vergleich mit der ausländischen Literatur nur bedingt möglich ist. Ebenfalls muss erwähnt werden, dass nur Leistungen untersucht werden, die über die Franchise hinaus abgerechnet werden. Somit wurden nur Leistungen erfasst, die sozialisierte Kosten verursacht haben; und es ist keine Aussage über die effektive Anzahl Konsultationen möglich. Als letzter Punkt muss bedacht werden, dass die Beobachtungsdauer auf ein Jahr beschränkt war. Es ist also nicht ersichtlich, ob die beobachteten Behandlungshäufigkeiten denjenigen vergangener oder folgender Jahre entsprechen.

\section{Schlussfolgerungen}

Mehr als jede dritte ambulante Behandlung für Patienten über 65 Jahre wird durch einen Hausarzt erbracht. In ländlichen Regionen gehen ältere Menschen bedeutend weniger häufig zum Arzt, werden aber öfter durch Hausärzte betreut als in städtischen Gebieten. Dies zeigt nicht nur, wie wichtig die Hausärzte vor allem in ländlichen Gebieten sind, sondern weist auch darauf hin, dass eine angemessene geriatrische Ausbildung der Haus- und Spezialärzte in Zukunft an Bedeutung gewinnen muss.

\section{Verdankungen}

Die vorliegende Arbeit ist Teil des Projekts «Démo Médicale» des Schweizerischen Gesundheitsobservatoriums (OBSAN). Ohne die Kooperation und Mitarbeit von Dr. H. Jaccard Ruedin des OBSAN und von M. Bertschi von santésuisse wäre die Arbeit nicht möglich gewesen.

\section{Literatur}

1 Besdine R, Boult C, Brangman S, Coleman EA, Fried LP, Gerety M, et al. Caring for older Americans: the future of geriatric medicine. J Am Geriatr Soc. 2005;53(6 Suppl):S245-56.

2 Iliescu ML, Zanoschi G. Population aging and public health. The active aging concept. Rev Med Chir Soc Med Nat Iasi. 2005;109(1):120-3.

3 Lagergren M. The systems of care for frail elderly persons: the case of Sweden. Aging Clin Exp Res. 2002;14(4):252-7.

4 Montero-Odasso M, Przygoda P, Redondo N, Adamson J, Kaplan R. Health care for older persons in Argentina: a country profile. J Am Geriatr Soc. 2004;52(10):1761-5.

5 Santos-Eggimann B. Evolution of the needs of older persons. Aging Clin Exp Res. 2002;14(4):287-92.

6 Schoenenberger AW, Stuck AE. Health care for older persons in Switzerland: a country profile. J Am Geriatr Soc. 2006;54(6):986-90.

7 Zanoschi G, Iliescu ML. Population aging. Current issues. Rev Med Chir Soc Med Nat Iasi. 2004; 108(1):61-5.

8 Mold JW, Green LA, Fryer GE. General internists and family physicians: partners in geriatric medicine? Ann Intern Med. 2003;139(7):594-6.

9 Probst JC, Moore CG, Baxley EG, Lammie JJ. Ruralurban differences in visits to primary care physicians. Fam Med. 2002;34(8):609-15.

10 Baldwin LM, Rosenblatt RA, Schneeweiss R, Lishner DM, Hart LG. Rural and urban physicians: does the content of their Medicare practices differ? J Rural Health. 1999;15(2):240-51.

11 Xakellis GC. Are patients who use a generalist physician healthier than those who seek specialty care directly? Fam Med. 2005;37(10):719-26.

12 Bisig B, Gutzwiller F (Hrsg.). Gesundheitswesen Schweiz: Gibt es Unter- oder Überversorgung? Zürich: Rüegger; 2004.

13 Schuler M, Dessemontet P, Joye D, Perlik M. Die Raumgliederung der Schweiz. Eidgenössische Volkszählung 2000. Neuenburg: Bundesamt für Statistik; 2005.

14 Jaccard Ruedin H, Roth M, Bétrisey C, Marzo N, Busato A. Offre et recours aux soins médicaux ambulatoires en Suisse. Neuenburg: Schweizerisches Gesundheitsobservatorium; 2007.

15 PANORAMA - Bevölkerung. Neuenburg: Bundesamt für Statistik; 2006.

16 Beschleunigte Alterung der Bevölkerung in den kommenden 30 Jahren. Medienmitteilung. Neuenburg: Bundesamt für Statistik; 2006.

17 Marty F. Wo eröffnen die Hausärzte heute ihre Praxis? PrimaryCare. 2006;6(9-10):181-3.

18 Hammer R. Stirbt der Hausarzt aus? PrimaryCare. 2005;5(28-29):628-9.

19 Hermann M, Heye C, Leuthold H, GIUZ. Soziokulturelle Unterschiede in der Schweiz - Vier Indizes zu räumlichen Disparitäten, 1990-2000. Neuenburg: Bundesamt für Statistik; 2005. 